\title{
System-level perturbations of cell metabolism using CRISPR/Cas9
}

Tadas Jakočiūnas $^{1}$, Michael K. Jensen ${ }^{1}$, Jay D. Keasling ${ }^{1-4}$

${ }^{1}$ The Novo Nordisk Foundation Center for Biosustainability, Technical University of Denmark, Denmark

${ }^{2}$ Joint BioEnergy Institute, Emeryville, CA, USA

${ }^{3}$ Biological Systems \& Engineering Division, Lawrence Berkeley National Laboratory, Berkeley, CA, USA

${ }^{4}$ Department of Chemical and Biomolecular Engineering \& Department of Bioengineering University of California, Berkeley, CA, USA

*Address correspondence to Michael K. Jensen: mije@biosustian.dtu.dk

\begin{abstract}
CRISPR/Cas9 (clustered regularly interspaced palindromic repeats and the associated protein Cas9) techniques have made genome engineering and transcriptional reprogramming studies more advanced and cost-effective. For metabolic engineering purposes, the CRISPR-based tools have been applied to single and multiplex pathway modifications and transcriptional regulations. The effectiveness of these tools allows researchers to implement genome-wide perturbations, test model-guided genome editing strategies, and perform transcriptional reprogramming perturbations in a more advanced manner than previously possible. In this mini-review we highlight recent studies adopting CRISPR/Cas9 for systems-level perturbations and model-guided metabolic engineering.
\end{abstract}

\section{Introduction}

CRISPR/Cas9 is revolutionizing biotechnology due to the speed and precision by which genomes and transcript abundances can be modified $\left[1^{\circ}, 2\right]$. Genome edits and transcriptional reprogramming which used to require years, non-natural selection pressure, and sequential transformation procedures, can now be completed in multiplex, and without the introduction of markers, within a week [3,4*0. In brief, CRISPR/Cas9, which originally was described as an adaptive immune system in bacteria and archaea [5-7], relies on the flexible guiding of the Cas9 nuclease by a short guide RNA (gRNA) to the genomic site of 
interest [8,9]. The guiding of Cas9, or nuclease deficient versions thereof (dCas9), has allowed the development of a variety of tools for integration of genes into chromosomes $[3,10-12]$, gene knock-outs $\left[13^{\circ}, 14^{\circ}-16\right]$, directed evolution experiments [17 $]$, epigenetic perturbations [18], and transcriptional regulation $\left[4^{* *}, 19^{*}, 20^{\circ}, 21^{\circ}\right]$. Indeed, CRISPR/Cas9 has been adapted and used in many different organisms for a variety of purposes [22] (Table 1 [16,23-37]), and most importantly, tools based on CRISPR have enabled inexpensive genetic manipulations in organisms which were previously troublesome, or even impossible, to engineer [27,31,32,34,37].

In relation to the design of cell factories for biobased production of chemicals, the CRISPR/Cas9 technologies enable researchers to perturb cellular metabolism more costeffectively. This includes facile integration of biosynthetic genes or whole pathways, limiting flux through competing pathways, (re-)direction of pathway intermediates and cofactors to the desired product, and engineering of enzymes with increased catalytic activity $\left[1^{\circ}\right]$. With such efficient tools at hand, metabolic engineers are confronted with an immense genome-wide solution space for improving cell factory performance. To assist genomeengineering decision-making, metabolic engineers are now starting to adopt i) systemslevel perturbations coupled with selection, and ii) metabolic models to guide the genomic edits and flux perturbations required for improving the production of a desired chemicals or pharmaceuticals. Both routes are mutually important, in order to rationalize the design of best-performing cell factories.

In this mini-review we first present the emerging applications where CRISPR/Cas9 technologies have been used for systems-level genome and metabolic engineering. Secondly, we present how dCas 9 has been used for balanced and model-guided transcriptional reprogramming of microbes, enabling metabolic models to leverage the CRISPR/Cas9 technology for experimental validation of model-guided designs. Lastly we put forward future perspectives on how CRISPR/Cas9 technology can further support the mapping of phenotypes onto genotypes, and thereby further support the refinement of metabolic models.

\section{CRISPR/Cas9 for systems-level genome and metabolic engineering}

To optimally design and engineer cell factories for increased production of chemicals, fuels and pharmaceuticals, data on the overall cellular states, chemical-genetic interactions, and metabolism should be well understood at the systems level. Nevertheless, even with extensive usage of a variety of omics approaches, many protein-coding gene functions, and 
how they are regulated, are still poorly understood. To overcome these issues and better understand them, genome-wide studies and large library screens are sought after (Figure 1). Perturbation of gene expression is an often sought after means to experimentally determine gene function. For this purpose, several studies have employed CRISPR/Cas9 for single gene repression/activation or knock-out $\left[38^{*}-40,41^{* *}, 42\right]$. Moreover, in order to infer conditional functionality, a recent study explored dCas9-mediated CRISPR interference to identify chemical-genetic interactions in yeast (Figure 2a) $\left[43^{* *}\right]$. Here, the authors tested a library of 989 unique gRNAs in the presence of various compounds known to inhibit growth, and from this determined both useful design rules for gRNAs and important genetic-chemical interactions [43*]. In another study, Cress and colleagues interrogated dCas9 repressive capabilities on a mutagenesis library of T7-lac promoter [44*0. This approach allowed generation of a set of inducible and variable-strength promoters, which could be regulated by both LacI and dCas9. Further, the authors designed and implemented selected promoters for throttling and selectively driving the violacein biosynthetic pathway in E. coli for targeted redirection of carbon flux. Using this synthetic promoter approach authors were able to increase production of proviolacein and deoxyviolacein more than tenfold compared to a reference design.

Beyond systematic single-gene targeting, mapping complex traits onto genotypes, requires systems-level methodologies to target genome-wide interactions between multiple genes. To tackle this, Perturb-Seq and CRISP-seq methods were recently outlined $\left[45^{\circ}, 46^{\circ}\right]$. These methods basically consists of combining the modularity of CRISPR/Cas9 to perform multi-locus (ie. delivery of multiple gRNAs in combination) gene perturbation with massively parallel single cell RNA sequencing as a genome-wide functional readout $\left[45^{*}, 46^{\circ}\right]$. From these methods it is possible to screen the transcriptional effects in several hundred thousands of single cell combinatorial gene knock-out events. In both cases, the methods allowed mapping phenotypes onto the identity of the perturbation, based on an expressed guide barcode. Though these studies were performed in mammalian cells and largely focused on developmental traits, the strategy of pooled gRNA screening and singlecell sequencing should be applicable to diverse cell types with a sequenced genome and amenable to genetic transformation. Likewise, Perturb-seq, but presumably also CRISPseq, is useful for dCas9-mediated genome-wide expression perturbations [47].

In another systems-level study of relevance to metabolic engineering, Garst et al., applied CRISPR-enabled trackable genome engineering (CREATE) to reverse engineer all 645 nonsynonymous mutations identified in an evolved thermotolerant E. coli strain [48*,49] 
(Figure 2b). Not only does this method demonstrate multi-loci editing at the genome scale, but since CREATE libraries mapping tens of thousands genome-wide mutations can be built, transformed and selected for by a single researcher in just 1-2 weeks, this method also significantly reduces the turnaround time of design-build-test cycles compared to month- and year-long adaptive laboratory evolution experiments.

Summing up, through cost-effective DNA synthesis and efficient multiplex CRISPR/Cas9 technologies, more and more gRNAs are experimentally tested and higher-order genetic interactions are being uncovered. This improves the predictive power for designing functional gRNAs, and should furthermore allow genome-wide screens to uncover optimal genotypes for desired metabolic states and phenotypic traits.

\section{Balanced metabolic engineering by CRISPR/dCas9}

As evidenced in the examples above, balancing expression of both heterologous and endogenous genes or pathways is of paramount importance, and dCas9-mediated transcriptional reprogramming is becoming a widely used technique in the fields of synthetic and systems biology (Figure 1). In this section we will review examples of dCas 9 applications for multiplex, systems-level and model-guided repression of gene expression in relation to metabolic engineering purposes.

Firstly, a recent study explored dCas 9 for balancing expression in the mevalonate biosynthetic pathway in E. coli for production of terpenoids [19*3. Here, the expression of target genes was tuned to minimize the production load, by applying L-rhamnose-inducible dCas9 for gene repression. The authors showed that the tool was capable of balancing expression of key enzymes, thereby increasing the production of selected terpenoids. Moreover, Li and colleagues explored dCas9-mediated repression of gene expressions responsible for cell growth in E. coli, and thereby developed genetic switches to increase production $\left[50^{\circ}\right]$. Here four genes were tested to decouple production from cell growth. Repression of each gene individually decreased the cell growth significantly and increased the production of mevalonate up to $41 \%$.

Similarly, by varying the proximity of gRNAs to the transcription start site, Deaner and Alper recently reported a method for systematic testing of enzyme perturbation sensitivities via graded dCas9 modulation in Saccharomyces cerevisiae (STEPS) [51]. Using this method, the authors identified rate limiting steps in glycerol biosynthesis pathway and optimized the pentose phosphate flux for xylose catabolism, ultimately resulting in a 7.8- 
fold increase in 3-dehydroshikimate titer and the identification of unique targets for xylose catabolism, respectively [ $\left[51^{\circ}\right]$.

Zalatan et al. have developed an RNA scaffold and dCas9-based system for gene activation and repression and applied it to balance expression of bacterial biosynthetic violacein pathway in yeast $S$. cerevisiae [4 $4^{\circ}$. This approach allowed for simultaneous activation of VioA and $\mathrm{VioC}$, and repression of $\mathrm{VioD}$ to achieve redirection of the product output [ $\left.4^{\circ}\right]$. Likewise, another recent study has developed a method called SWITCH, which also allows gene transcription activation and repression in yeast $S$. cerevisiae [21 ${ }^{\circ}$. Here, the authors demonstrated the usability of dCas9-based systems for activation of the ADE2 gene expressed from the GAL2 promoter and repression of the TSC13 gene to achieve an increased production of naringenin $\left[21^{\circ}\right]$.

Finally, Cress et al. have been the first to report model-guided metabolic engineering in $E$. coli to predict multiple dCas9 gene targets supporting improved chemical production. In this study, the authors combined OptForce [52] and CRISPR arrays for repressing three enzyme-encoding genes and thereby improving the production of the plant flavonoid naringenin by 2,5 fold [53 $\left.{ }^{\circ}\right]$ (Figure $2 \mathrm{c}$ ).

In relation to metabolic engineering, dCas9 was recently adopted for photoautotrophic cyanobacteria - a promising organism for production of biofuels and other valuable chemicals [54]. In a first study, dCas9 was employed for repressing the formation of the carbon storage compounds polyhydroxybutyrate (PHB) and glycogen [55]. A 10-fold repression of PHB was achieved by targeting phaE gene, encoding polyhydroxyalkanoate synthase, which was proposed to result in higher abundance of acetyl-CoA, a key precursor for many chemicals. Similarly, by repressing $g \operatorname{lgC}$ gene, encoding ADP-glucose pyrophosphorylase, the glycogen accumulation was reduced by $25 \%$, thereby providing cells with additional carbon during starvation. In another study, the authors explored dCas9's ability to repress $g \ln A$, encoding glutamine synthetase I, for redirection of central carbon flux. Through this, lactate production was improved 2-fold [56 ${ }^{\circ}$.

In addition to growth, cell morphology or shape can also have a profound effect on chemical production. Elhadi et al. investigated effects of dCas9 controlled gene repression affecting cell morphology for production of PHB [57]. The genes fts $Z$ and $m r e B$, encoding bacterial fission ring and skeletons, were down-regulated to achieve longer and larger $E$. coli cells, with $13 \%$ to $36 \%$ increased capacity to accumulate PHB's. 
In summary, dCas9 has been shown to be efficient and promising tool for metabolic engineering purposes, and it has been applied in various organisms for balancing expression of genes and both endogenous and heterologous pathways.

\section{Perspectives and conclusion}

The emerging studies on systems-level genome engineering, as evidenced by the use of Perturb-Seq, CRISP-Seq and CREATE, address the need for an improved understanding of higher-order genetic interactions in order to enable the mapping of phenotypes onto genotypes $\left[45^{*}, 46^{*}, 48^{*}\right]$. These technologies allow for genome-scale readouts in the form of next-generation sequencing in relation to essentially any environmental condition or selection pressure of interest. Moreover, as the methods use DNA as input, they easily scale with both the cost of DNA synthesis and sequencing. In addition to the technical advances offered by these methods, we envision that systems-level genome and metabolic engineering will be useful for validating metabolic models by experimentally mapping genome-wide genetic interactions and transcript perturbations to phenotypes of interest. Though not targeting massive genetic interactions, the experimental validation of modelguided OptForce outputs by Cress et al. perfectly exemplifies this emerging trend [53*0]. Vice versa, we envision that access to experimental data from systems-level engineering efforts should allow refinement of genome-scale metabolic models.

Also, we envision that transcriptional regulation driven by dCas9 will be used more and more to elucidate complicated metabolic pathways, balance the dosage of key enzymes and point out bottlenecks arising when heterologous pathways are expressed. In addition, it can be used to understand gene-to-gene relations to uncover inhibitory or activating effects of enzymes by up- or down-regulation of gene expression. Importantly, dCas9's ability to regulate multiple targets resembles native transcriptional reprogramming and thereby allows for testing and redirecting metabolic states with much higher impact compared to earlier single-gene regulatory control mechanisms.

Finally, just as for Cas9-mediated knock-out and knock-in studies, we also envision that dCas9 will become widely used tool to elucidate and improve model-guided predictions. Here special attention could be given to kinetic metabolic modeling [58], ultimately enabling predictions of overall cell factory productivity in relation to a pathway's enzyme expression levels.

\section{Acknowledgements}


This work was funded by the Novo Nordisk Foundation and by Joint BioEnergy Institute (http://www.jbei.org/), which is supported by the US Department of Energy, Office of Science, Office of Biological and Environmental Research, through contract DE-AC0205CH11231 between Lawrence Berkeley National Laboratory and the US Department of Energy.

\section{References and recommended reading}

Papers of particular interest, published within the period of review, have been highlighted as:

- of special interest

•• of outstanding interest

\section{References}

1. Jakočiūnas T, Jensen MK, Keasling JD: CRISPR/Cas9 advances engineering of microbial cell factories. Metab. Eng. 2016, 34:44-59.

This review provides an overview of the CRISPR/Cas9 tools available for model organisms E.coli and S.cerevisiae.

2. Khatodia S, Bhatotia K, Passricha N, Khurana SMP, Tuteja N: The CRISPR/Cas Genome-Editing Tool: Application in Improvement of Crops. Front. Plant Sci. 2016, 7:506.

3. Jakočiūnas T, Rajkumar AS, Zhang J, Arsovska D, Rodriguez A, Jendresen CB, Skjødt ML, Nielsen AT, Borodina I, Jensen MK, et al.: CasEMBLR: Cas9-Facilitated Multiloci Genomic Integration of in Vivo Assembled DNA Parts in Saccharomyces cerevisiae . ACS Synth. Biol. 2015, 4:1226-1234.

4*. Zalatan JG, Lee ME, Almeida R, Gilbert LA, Whitehead EH, La Russa M, Tsai JC, Weissman JS, Dueber JE, Qi LS, et al.: Engineering Complex Synthetic Transcriptional Programs with CRISPR RNA Scaffolds. Cell 2014, 160:339-50. Here dCas9 was used for repression and activation of genes in yeast and human cells, but instead of dCas9 being fused with activating or represing domains, gRNA scaffolds were design to contain binding sites for recruitment of effector proteins.

5. Bolotin A, Quinquis B, Sorokin A, Dusko Ehrlich S: Clustered regularly interspaced short palindrome repeats (CRISPRs) have spacers of extrachromosomal origin. Microbiology 2005, 151:2551-61.

6. Mojica FJM, Díez-Villaseñor C, García-Martínez J, Soria E: Intervening sequences of 
regularly spaced prokaryotic repeats derive from foreign genetic elements. $J$. Mol. Evol. 2005, 60:174-82.

7. Pourcel C, Salvignol G, Vergnaud G: CRISPR elements in Yersinia pestis acquire new repeats by preferential uptake of bacteriophage DNA, and provide additional tools for evolutionary studies. Microbiology 2005, 151:653-63.

8. Jinek M, Chylinski K, Fonfara I, Hauer M, Doudna JA, Charpentier E: A Programmable Dual-RNA-Guided DNA Endonuclease in Adaptive Bacterial Immunity. Science (80). 2012, 337.

9. Gasiunas G, Barrangou R, Horvath P, Siksnys V: Cas9-crRNA ribonucleoprotein complex mediates specific DNA cleavage for adaptive immunity in bacteria. Proc. Natl. Acad. Sci. 2012, 109:E2579-E2586.

10. Jessop-Fabre MM, Jakočiūnas T, Stovicek V, Dai Z, Jensen MK, Keasling JD, Borodina I: EasyClone-MarkerFree: A vector toolkit for marker-less integration of genes into Saccharomyces cerevisiae via CRISPR-Cas9. Biotechnol. J. 2016, 11:1110-7.

11. Jiang W, Bikard D, Cox D, Zhang F, Marraffini LA: RNA-guided editing of bacterial genomes using CRISPR-Cas systems. Nat. Biotechnol. 2013, 31:233-9.

12. Lee JS, Kallehauge TB, Pedersen LE, Kildegaard HF: Site-specific integration in CHO cells mediated by CRISPR/Cas9 and homology-directed DNA repair pathway. Sci. Rep. 2015, 5:8572.

13. Jakočiūnas T, Bonde I, Herrgård M, Harrison SJ, Kristensen M, Pedersen LE, Jensen MK, Keasling JD: Multiplex metabolic pathway engineering using CRISPR/Cas9 in Saccharomyces cerevisiae. Metab. Eng. 2015, 28C:213-22.

Multiplex CRISPR/Cas9 system was established where up to 5 gRNAs were expressed on a single plasmid. System was applied for metabolic engineering purpose to increase mevalonate production in yeast.

14. Li Y, Lin Z, Huang C, Zhang Y, Wang Z, Tang Y, Chen T, Zhao X: Metabolic engineering of Escherichia coli using CRISPR-Cas9 meditated genome editing. Metab. Eng. 2015, 31:13-21.

This paper describes development of CRISPR/Cas9 system for E.coli. Genome engineering tools were used to integrate $\beta$-carotene synthetic pathway, and for optimization of both methylerythritol-phosphate (MEP) and central metabolic pathways.

15. Pyne ME, Moo-Young M, Chung DA, Chou CP: Coupling the CRISPR/Cas9 system 
to lambda Red recombineering enables simplified chromosomal gene replacement in Escherichia coli. Appl. Environ. Microbiol. 2015, 81:5103-14.

16. Grav LM, Lee JS, Gerling S, Beuchert Kallehauge T, Holmgaard Hansen A, Kol S, Lee GM, Ebdrup Pedersen L, Faustrup Kildegaard H: One-step generation of triple knockout CHO cell lines using CRISPR Cas9 and fluorescent enrichment. Biotechnol. J. 2015, 10:1446-56.

17. Hess GT, Frésard L, Han K, Lee CH, Li A, Cimprich KA, Montgomery SB, Bassik MC: Directed evolution using dCas9-targeted somatic hypermutation in mammalian cells. Nat. Methods 2016, 12:1036-42.

This paper describes a method based on Cas9 for directed evolution of proteins in mamalian cells.

18. Hilton IB, D'Ippolito AM, Vockley CM, Thakore PI, Crawford GE, Reddy TE, Gersbach CA: Epigenome editing by a CRISPR-Cas9-based acetyltransferase activates genes from promoters and enhancers. Nat. Biotechnol. 2015, 33:510-7.

19“. Kim SK, Han GH, Seong W, Kim H, Kim S-W, Lee D-H, Lee S-G: CRISPR interference-guided balancing of a biosynthetic mevalonate pathway increases terpenoid production. Metab. Eng. 2016, 38:228-40.

$\mathrm{dCas} 9$ based method for expression balancing of mevalonate pathway to increase production of isoprenoids.

20. Gilbert LA, Larson MH, Morsut L, Liu Z, Brar GA, Torres SE, Stern-Ginossar N, Brandman O, Whitehead EH, Doudna JA, et al.: CRISPR-mediated modular RNAguided regulation of transcription in eukaryotes. Cell 2013, 154:442-51.

Among the first ones to show that dCas9 can be fused with transcription activating or represing domains and when guided to the gene of interest can activate or repress those genes. The new tool has been tested in human and yeast cells.

21․ Vanegas KG, Lehka BJ, Mortensen UH: SWITCH: a dynamic CRISPR tool for genome engineering and metabolic pathway control for cell factory construction in Saccharomyces cerevisiae. Microb. Cell Fact. 2017, 16:25.

Have developed a Cas9/dCas9 based system that integrates both genetic engineering and transcriptional control of pathways into one system.

22. Hsu PD, Lander ES, Zhang F: Development and Applications of CRISPR-Cas9 for Genome Engineering. Cell 2014, 157:1262-78.

23. Lee JS, Kallehauge TB, Pedersen LE, Kildegaard HF, Noh SM, Sathyamurthy M, Lee GM, Wurm FM, Wilson C, Bellen HJ, et al.: Site-specific integration in CHO cells 
mediated by CRISPR/Cas9 and homology-directed DNA repair pathway. Sci. Rep. 2015, 5:8572.

24. Ronda C, Pedersen LE, Hansen HG, Kallehauge TB, Betenbaugh MJ, Nielsen AT, Kildegaard HF: Accelerating genome editing in CHO cells using CRISPR Cas9 and CRISPy, a web-based target finding tool. Biotechnol. Bioeng. 2014, 111:160416.

25. Cobb RE, Wang Y, Zhao H: High-Efficiency Multiplex Genome Editing of Streptomyces Species Using an Engineered CRISPR/Cas System. ACS Synth. Biol. 2015, 4:723-8.

26. Huang H, Zheng G, Jiang W, Hu H, Lu Y: One-step high-efficiency CRISPR/Cas9mediated genome editing in Streptomyces. Acta Biochim. Biophys. Sin. (Shanghai). 2015, 47:231-43.

27. Tong Y, Charusanti P, Zhang L, Weber T, Lee SY: CRISPR-Cas9 Based Engineering of Actinomycetal Genomes. ACS Synth. Biol. 2015, doi:10.1021/acssynbio.5b00038.

28. Wang Y, Zhang Z-T, Seo S-O, Choi K, Lu T, Jin Y-S, Blaschek HP: Markerless chromosomal gene deletion in Clostridium beijerinckii using CRISPR/Cas9 system. J. Biotechnol. 2015, 200:1-5.

29. Huang H, Chai C, Li N, Rowe P, Minton NP, Yang S, Jiang W, Gu Y: CRISPR/Cas9Based Efficient Genome Editing in Clostridium ljungdahlii, an Autotrophic GasFermenting Bacterium. ACS Synth. Biol. 2016, 12:1355-61

30. Oh J-H, van Pijkeren J-P: CRISPR-Cas9-assisted recombineering in Lactobacillus reuteri. Nucleic Acids Res. 2014, 42:e131.

31. Nødvig CS, Nielsen JB, Kogle ME, Mortensen UH: A CRISPR-Cas9 System for Genetic Engineering of Filamentous Fungi. PLoS One 2015, 10:e0133085.

32. Liu R, Chen L, Jiang Y, Zhou Z, Zou G: Efficient genome editing in filamentous fungus Trichoderma reesei using the CRISPR/Cas9 system. Cell Discov. 2015, 1:15007.

33. Jacobs JZ, Ciccaglione KM, Tournier V, Zaratiegui M: Implementation of the CRISPR-Cas9 system in fission yeast. Nat. Commun. 2014, 5:1-5.

34. Schwartz CM, Hussain MS, Blenner M, Wheeldon I: Synthetic RNA Polymerase III Promoters Facilitate High-Efficiency CRISPR-Cas9-Mediated Genome Editing in Yarrowia lipolytica. ACS Synth. Biol. 2016, 5:356-9.

35. Weninger A, Hatzl A-M, Schmid C, Vogl T, Glieder A: Combinatorial optimization 
of CRISPR/Cas9 expression enables precision genome engineering in the methylotrophic yeast Pichia pastoris. J. Biotechnol. 2016, 235:139-49.

36. Wendt KE, Ungerer J, Cobb RE, Zhao H, Pakrasi HB: CRISPR/Cas9 mediated targeted mutagenesis of the fast growing cyanobacterium Synechococcus elongatus UTEX 2973. Microb. Cell Fact. 2016, 15:115.

37. Li H, Shen CR, Huang C-H, Sung L-Y, Wu M-Y, Hu Y-C: CRISPR-Cas9 for the genome engineering of cyanobacteria and succinate production. Metab. Eng. 2016, 38:293-302.

38“. Gilbert L a, Horlbeck M a, Adamson B, Villalta JE, Chen Y, Whitehead EH, Guimaraes C, Panning B, Ploegh HL: Resource Genome-Scale CRISPR-Mediated Control of Gene Repression and Activation. Cell 2014, 159:647-61.

Among the first ones to show genome-wide screening with dCas9 for activating and repressing genes in mamalian cells.

39. Kiessling MK, Schuierer S, Stertz S, Beibel M, Bergling S, Knehr J, Carbone W, de Vallière $\mathrm{C}$, Tchinda $\mathrm{J}$, Bouwmeester $\mathrm{T}$, et al.: Identification of oncogenic driver mutations by genome-wide CRISPR-Cas9 dropout screening. BMC Genomics 2016, 17:723.

40. Bassett AR, Kong L, Liu J-L: A Genome-Wide CRISPR Library for HighThroughput Genetic Screening in Drosophila Cells. J. Genet. Genomics 2015, 42:301-9.

41‥ Koike-Yusa H, Li Y, Tan E-P, Velasco-Herrera MDC, Yusa K: Genome-wide recessive genetic screening in mammalian cells with a lentiviral CRISPR-guide RNA library. Nat. Biotechnol. 2013, 32:267-73.

Among the first ones to perform genome-wide knock-out screen in mammalian cells by using CRISPR/Cas9. The screen identified 27 known and 4 unknown genes that play a role in resistance to Clostridium septicum alpha-toxin or 6-thioguanine.

42. Shalem O, Sanjana NE, Hartenian E, Shi X, Scott DA, Mikkelsen TS, Heckl D, Ebert BL, Root DE, Doench JG, et al.: Genome-Scale CRISPR-Cas9 Knockout Screening in Human Cells. Science. 2014, 343.

43“. Smith JD, Suresh S, Schlecht U, Wu M, Wagih O, Peltz G, Davis RW, Steinmetz LM, Parts L, St.Onge RP, et al.: Quantitative CRISPR interference screens in yeast identify chemical-genetic interactions and new rules for guide RNA design. Genome Biol. 2016, 17:45. 
This paper determines rules for choosing efficient guiding gRNAs. Employing dCas9 and modelling approach authors identify that guides targeting low nucleosome occupancy regions and regions between the transcription start site (TSS) and $200 \mathrm{bp}$ upstream of the TSS are most effective.

44. Cress BF, Jones JA, Kim DC, Leitz QD, Englaender JA, Collins SM, Linhardt RJ, Koffas MAG: Rapid generation of CRISPR/dCas9-regulated, orthogonally repressible hybrid T7-lac promoters for modular, tuneable control of metabolic pathway fluxes in Escherichia coli. Nucleic Acids Res. 2016, 44:4472-85.

CRISPR array system based on dCas9 for down regulation of genes in E.coli Authors show that down-regulating novel and computationally predicted metabolic engineering targets can improve the yield of certain compound.

45*. Dixit A, Parnas O, Li B, Chen J, Fulco CP, Jerby-Arnon L, Marjanovic ND, Dionne D, Burks T, Raychowdhury R, et al.: Perturb-Seq: Dissecting Molecular Circuits with Scalable Single-Cell RNA Profiling of Pooled Genetic Screens. Cell 2016, 167:1853-66.

Has developed a method called Perturb-seq, which combines singlecell RNA sequencing (RNA-seq) and CRISPR-based perturbations to perform many pooled assays.

46“. Jaitin DA, Weiner A, Yofe I, Lara-Astiaso D, Keren-Shaul H, David E, Salame TM, Tanay A, Oudenaarden A van, Amit I: Dissecting Immune Circuits by Linking CRISPR-Pooled Screens with Single-Cell RNA-Seq. Cell 2016, 167:1883-96.

Has developed a method called CRISP-seq, which integrates single-cell RNA sequencing and CRISPR-pooled screens.

47. Adamson B, Norman TM, Jost M, Cho MY, Nuñez JK, Chen Y, Villalta JE, Gilbert LA, Horlbeck MA, Hein MY, et al.: A Multiplexed Single-Cell CRISPR Screening Platform Enables Systematic Dissection of the Unfolded Protein Response. Cell 2016, 167:1867-82.

48“. Garst AD, Bassalo MC, Pines G, Lynch SA, Halweg-Edwards AL, Liu R, Liang L, Wang Z, Zeitoun R, Alexander WG, et al.: Genome-wide mapping of mutations at single-nucleotide resolution for protein, metabolic and genome engineering. Nat. Biotechnol. 2016, 35:48-55.

Has developed CRISPR based method for trackable genome engineering (CREATE) and reverse engineered all 645 nonsynonymous mutations identified in an evolved thermotolerant E. coli strain. 
49. Tenaillon O, Rodríguez-Verdugo A, Gaut RL, McDonald P, Bennett AF, Long AD, Gaut BS: The Molecular Diversity of Adaptive Convergence. Science. 2012, 335.

50. Li S, Jendresen CB, Grünberger A, Ronda C, Jensen SI, Noack S, Nielsen AT: Enhanced protein and biochemical production using CRISPRi-based growth switches. Metab. Eng. 2016, 38:274-84.

Have showed that CRISPR/Cas9 can be used to regulate expression of genes that are responsible for groth and by slowing the groth increase production proteins and chemicals.

51․ Deaner M, Alper HS: Systematic Testing of Enzyme Perturbation Sensitivities via Graded dCas9 Modulation in Saccharomyces cerevisiae. Metab. Eng. 2017, doi:10.1016/j.ymben.2017.01.012.

This paper describes a method for systematic testing of enzyme perturbation sensitivities via graded dCas9 modulation in Saccharomyces cerevisiae.

52. Ranganathan S, Suthers PF, Maranas CD: OptForce: An Optimization Procedure for Identifying All Genetic Manipulations Leading to Targeted Overproductions. PLoS Comput. Biol. 2010, 6:e1000744.

53“. Cress BF, Toparlak ÖD, Guleria S, Lebovich M, Stieglitz JT, Englaender JA, Jones JA, Linhardt RJ, Koffas MAG: CRISPathBrick: Modular Combinatorial Assembly of Type II-A CRISPR Arrays for dCas9-Mediated Multiplex Transcriptional Repression in E. coli. ACS Synth. Biol. 2015, 4:987-1000.

CRISPR array system based on dCas9 for down regulation of genes in E.coli Authors show that down-regulating novel and computationally predicted metabolic engineering targets can improve the yield of certain compound.

54. Angermayr SA, Gorchs Rovira A, Hellingwerf KJ, Ducat DC, al. et, Wijffels RH, al. et, Keasling JD, Berg JJ, al. et, et al.: Metabolic engineering of cyanobacteria for the synthesis of commodity products. Trends Biotechnol. 2015, 33:352-61.

55. Yao L, Cengic I, Anfelt J, Hudson EP: Multiple Gene Repression in Cyanobacteria Using CRISPRi. ACS Synth. Biol. 2016, 5:207-12.

56. Gordon GC, Korosh TC, Cameron JC, Markley AL, Begemann MB, Pfleger BF: CRISPR interference as a titratable, trans-acting regulatory tool for metabolic engineering in the cyanobacterium Synechococcus sp. strain PCC 7002. Metab. Eng. 2016, 38:170-9. 
This study shows usability of dCas 9 for gene repression in cyanobacteria to increase central carbon flux. Such experimental design provides 2-fold increase in lactate production.

57. Elhadi D, Lv L, Jiang X-R, Wu H, Chen G-Q: CRISPRi engineering E. coli for morphology diversification. Metab. Eng. 2016, 38:358-69.

58. Theisen MK, Lafontaine Rivera JG, Liao JC: Stability of Ensemble Models Predicts Productivity of Enzymatic Systems. PLOS Comput. Biol. 2016, 12:e1004800.

\section{Figure Legends}

\section{Figure 1. CRISPR/Cas9 usability for metabolic engineering purposes.}

Usability of CRISPR/Cas9 for metabolic engineering purposes is depicted in the figure, showing its wide application in metabolic pathway engineering and genome editing in general, genome-wide and large library screening as well as transcriptional regulation of pathway or competing pathway genes and overall combinatorial expression balancing of metabolic pathways. GOI - gene of interest; HR - homologous recombination; dsOligo double stranded oligo.

Figure 2. CRISPR/Cas9 usability for model-guided and library screening approaches (a) dCas9 fused with Mxi1 and inducible gRNA expression system was used for screening the gRNA library targeting 4 genes to determine rules for best gRNA design. Screen was used to test chemical-genetic interaction, which showed that silencing of 4 genes in the presence of different small molecule inhibitors resulted in decreased cellular growth [43*]. (b). CREATE cassettes, containing gRNA and homology arm used for integration by homologous recombination, are used for protein engineering, reconstruction of adaptive laboratory evolution experiments and identification of stress tolerance and antibiotic resistance genes in bacteria. Experimental setup consists of: in silico design and synthesis of oligos (CREATE cassettes), subsequent PCR amplification and multiplex cloning of editing vectors, vectors are transformed to the cells and after selection, frequency before and after selection is determined by sequencing [48*0] (c) Model-guided approach was used to predict targets for expressional down-regulation of genes for increased naringenin production. CRISPR array was used to achieve transcriptional silencing of 3 genes simultaneously, which resulted in 2.5-fold increase in naringenin production [53“*]. 